and Pericardial Diseases. Eur Heart J. 2013;34:2636-48, 2648a-2648d.

7. Torres JR, Castro JS, Rodríguez L, Saravia V, Arvelaez J, Ríos-Fabra A, et al. Chikungunya fever: ftypical and lethal cases in the Western Hemisphere: A Venezuelan experience. IDCases. 2014;2:6-10. http://dx.doi.org/10.1016/j.idcr.2014.12.002

8. Rollé A, Schepers K, Cassadou S, Curlier E, Madeux B, Hermann-Storck C, et al. Severe sepsis and septic shock associated with chikungunya virus infection, Guadeloupe, 2014. Emerg Infect Dis. 2016;22:891-4. http://dx.doi.org/10.3201/eid2205.151449

9. Cao-Lormeau V-M, Blake A, Mons S, Lastère S, Roche C, Vanhomwegen J, et al. Guillain-Barré syndrome outbreak associated with Zika virus infection in French Polynesia: a casecontrol study. Lancet. 2016;387:1531-9. http://dx.doi.org/10.1016/ S0140-6736(16)00562-6

Address for correspondence: Adrien Koeltz, Department of Anesthesiology and Intensive Care, Centre Hospitalier Universitaire Bichat Claude-Bernard, 46 Rue Henri Huchard, 75018 Paris, France; email: adrien.koeltz@aphp.fr

\section{African Swine Fever Virus, Siberia, Russia, 2017}

\section{Denis Kolbasov, llya Titov, Sodnom Tsybanov, Andrey Gogin, Alexander Malogolovkin}

Author affiliation: Federal Research Center for Virology and Microbiology, Pokrov, Russia

DOI: https://doi.org/10.3201/eid2404.171238

African swine fever (ASF) is arguably the most dangerous and emerging swine disease worldwide. ASF is a serious problem for the swine industry. The first case of ASF in Russia was reported in 2007. We report an outbreak of ASF in Siberia, Russia, in 2017.

A frican swine fever (ASF) is arguably the most dangerous swine disease worldwide. ASF virus (ASFV) is highly virulent for domestic swine and remains a global threat because no effective vaccine is available to eradicate the disease. The emergent potential of ASF has been demonstrated by its spread into Russia. In the 10 years since ASF was first diagnosed in the Caucasian region of Russia (1), the disease has reached Palearctic regions and is spreading into western Europe $(2,3)$.

In 2017, the Federal Service for Veterinary and Phytosanitary Surveillance (Rosselkhoznadzor) reported that, during 2007-2017, >1,000 ASF outbreaks resulted in deaths of $\approx 800,000$ pigs in 46 regions across Russia (4). Production of backyard swine industry decreased by almost half, from 1,119 tons of pork in 2007 to 608 tons of pork in 2017 (5). However, highly industrialized pig farms showed increased production every year during this same period, despite the ASF epidemic.

ASF has seriously affected and is actively spread by wild boar populations in Russia, but accurate numbers of boar killed by ASF or culling attempts are difficult to estimate. In June 2017, ASF was detected in the Czech Republic in 2 wild boar ( 6 ), demonstrating disease spread toward western Europe. In 2017, ASFV cases among backyard domestic pigs were detected in July in Romania (7), and later in October 2017 in Moldova (8). We report an outbreak of ASF in Far Eastern Russia.

Early in March 2017, an ASF outbreak was reported on 1 backyard farm in the Irkutsk region near the border with Mongolia (Figure) (5). All pigs had clinical signs typical of acute ASF, and 40 pigs died within 6 days of the appearance of the first clinical signs. In a $5-\mathrm{km}$ risk zone established around the affected farm, 1,327 pigs were slaughtered within 3 days. Epidemiologic analysis showed that the farmer used table leftovers to feed pigs.

ASFV DNA was identified by real-time PCR in the frozen pork products found on the farm. The origin of contaminated pork products is still under investigation. It is likely that ASFV-contaminated pork products provided a source of infection because these products are the most common source of ASF infection on backyard farms (9). ASF outbreaks nearest to the outbreak in Irkutsk occurred $>4,000 \mathrm{~km}$ away in European Russia. Such a long geographic distance between ASF outbreaks within the country demonstrates that ASFV has a tremendous capacity for transboundary and transcontinental spread.

We identified the ASFV isolate from Irkutsk (ASFV/ Irkutsk/dom/2017) by using nucleotide sequencing and molecular analysis. This isolate has capsid protein $\mathrm{P} 72$ genotype II and central variable region I and is an intergenic region (IGR) I variant (GenBank accession nos. KY963545, KY938010, and KY982843, respectively) according to the nomenclature of Gallardo et al. (10). The intergenic region between the $I 73 R$ and $I 329 L$ genes at the right end of the ASFV/Irkutsk/dom/2017 genome contains no additional tandem-repeat sequences. The ASFV IGRI variant is identical to the ASFV/Georgia/wb/2007 index isolate of the epidemic in Georgia in 2007 but represents an ASFV variant that is rare among recent ASFV isolates in Russia. In comparison, all recent ASF outbreaks in European Russia and eastern Europe have been caused by ASFV of the IGRII variant, which has an insertion of a tandem-repeat sequence in the intergenic region between the I173R and the I329L protein genes. 


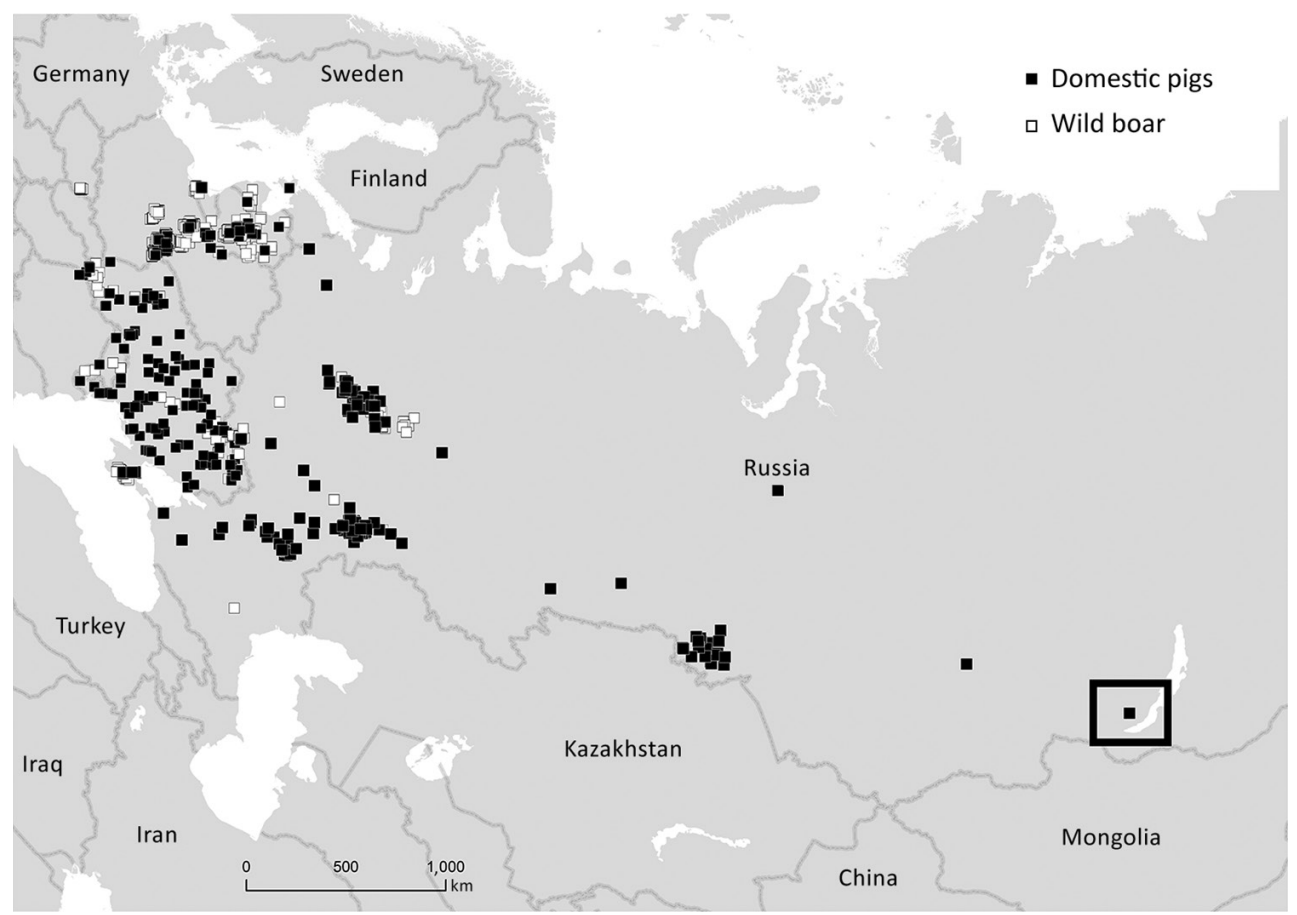

Figure. African swine fever outbreaks in Russia and countries in eastern Europe, 2017. Black box indicates outbreak in the Irkutsk region in Siberia, Russia.

Our results indicate that an ASF outbreak in Siberia in 2017 was caused by the pan-Russian strain of ASFV (genotype II, central variable region I, and IGRI) that contains B646L (P72), B602L, and intergenic region I173-I329L sequences identical to those of ASFV index isolate ASFV/Georgia/wb/2007 (GenBank accession no. FR682468.1). ASFV-contaminated pork products still pose a major risk for transboundary emergence and spread of ASF. ASFV/Irkutsk/dom/2017 is a highly virulent strain and causes acute ASF in domestic swine. Since the outbreak in Irkutsk, subsequent ASF outbreaks have occurred in Siberia (March-October 2017) and near the border with China, raising concerns that ASF might be introduced into a population of 500 million pigs. This continued and far-reaching spread of ASF in Russia demonstrates the threat of disease emergence and increased spread worldwide.

\section{Acknowledgment}

We thank Edan R. Tuman for reviewing the manuscript and providing useful comments.

This study was supported by the Federal Agency of Scientific Organizations (agreement no. 0615-2017-0001).

\section{About the Author}

Dr. Kolbasov is director of the Molecular Virology Unit, Federal Research Center for Virology and Microbiology, Pokrov, Russia. His research interests are epidemiology of emerging animal diseases, diagnostics, and vaccine development.

\section{References}

1. Gogin A, Gerasimov V, Malogolovkin A, Kolbasov D. African swine fever in the North Caucasus region and the Russian Federation in years 2007-2012. Virus Res. 2013;173:198-203. http://dx.doi.org/10.1016/j.virusres.2012.12.007

2. Śmietanka K, Woźniakowski G, Kozak E, Niemczuk K, Frączyk M, Bocian Ł, et al. African swine fever epidemic, Poland, 2014-2015. Emerg Infect Dis. 2016;22:1201-7. http://dx.doi.org/ 10.3201/eid2207.151708

3. Sánchez-Vizcaíno JM, Mur L, Martínez-López B. African swine fever (ASF): five years around Europe. Vet Microbiol. 2013;165:45-50. http://dx.doi.org/10.1016/j.vetmic.2012.11.030

4. The Federal Service for Veterinary and Phytosanitary Supervision [in Russian] [cited 2018 Feb 5]. http://www.fsvps.ru

5. Kovalev YI. Veterinary medicine and pigs. Swine production in Russia 2015-2020: current challenges, risks and solutions. Novosibirsk, May 18-19, 2017 [cited 2018 Feb 5]. http://www.pigproducer.net/uploads/media/3_Jurij-Kovalev_.pdf

6. World Organisation for Animal Health. World Animal Health Information Database. Exceptional epidemiological events [cited 2018 Feb 5]. http://www.oie.int/wahis_2/public/wahid.php/ Countryinformation/Countryreports

7. World Organisation for Animal Health. World Animal Health Information Database, African swine fever, Romania [cited $2018 \mathrm{Feb} 5]$. http://www.oie.int/wahis_2/public/wahid.php/ Reviewreport/Review?reportid $=24456$

8. World Organisation for Animal Health. World Animal Health Information Database. African swine fever, Moldava [cited 2018 Feb 5]. https://www.oie.int/wahis_2/public/wahid.php/ Reviewreport/Review?page_refer=MapFullEventReport\&report $\mathrm{id}=25134$

9. Food and Agricultural Organization of the United Nations. African swine fever in the Russian Federation: risk factors for Europe and beyond. Rome: The Organization; 2013.

10. Gallardo C, Fernández-Pinero J, Pelayo V, Gazaev I, Markowska-Daniel I, Pridotkas G, et al. Genetic variation among African swine fever genotype II viruses, eastern and central 
Europe. Emerg Infect Dis. 2014;20:1544-7. http://dx.doi.org/ 10.3201/eid2009.140554

Address for correspondence: Alexander Malogolovkin, Federal Research Center for Virology and Microbiology, 601125, Vladimirskaya Oblast', Pokrov, Russia; email: malogolovkin@inbox.ru

\section{Classical Swine Fever Outbreak after Modified Live LOM Strain Vaccination in Naive Pigs, South Korea}

\section{Sang H. Je, ${ }^{1}$ Taeyong Kwon, ${ }^{1}$ Sung J. Yoo, Dong-Uk Lee, SeungYoon Lee, Juergen A. Richt, Young S. Lyoo}

Author affiliations: Konkuk University, Seoul, South Korea (S.H. Je, T. Kwon, S.J. Yoo, D.-U. Lee, Y.S. Lyoo); HanByol Farm Tech, Namyangju, South Korea (S. Lee); Kansas State University, Manhattan, Kansas, USA (J.A. Richt)

DOI: https://doi.org/10.3201/eid2404.171319

We report classical swine fever outbreaks occurring in naive pig herds on Jeju Island, South Korea, after the introduction of the LOM vaccine strain. Two isolates from sick pigs had $>99 \%$ identity with the vaccine stain. LOM strain does not appear safe; its use in the vaccine should be reconsidered.

$\mathrm{C}$ lassical swine fever is a highly contagious disease of pigs that tremendously affects the swine industry. Although several countries have become free from classical swine fever after eradication programs, sporadic outbreaks continue to occur in most major pig-producing countries, and classical swine fever is endemic to some countries in Asia. Vaccination is regarded as one of the most effective tools to prevent and control classical swine fever. Modified live vaccines (MLVs) mainly containing C-strain have been used widely because of their safety and provide complete protection against virus challenge $(1,2)$.

Since 1974, the LOM strain has been the MLV strain for classical swine fever in South Korea. As a result of the government's classical swine fever eradication program, Jeju Island, South Korea, became a classical swine fever virus (CSFV)-free area, and vaccination efforts ceased there in 1999 (3). Strong prohibition of live pig trade has also contributed to the maintenance of CSFV-naive herds on Jeju Island for over a decade, although sporadic classical swine fever outbreaks have occurred in mainland South Korea, despite mandatory vaccination with the LOM strain (4). This study describes classical swine fever outbreaks in naive pig herds on Jeju Island caused by the MLV.

Since 2014, multiple classical swine fever outbreaks have occurred on Jeju Island (online Technical Appendix Figure 1, https://wwwnc.cdc.gov/EID/article/24/4/17-1319Techapp1.pdf). Clinical manifestation is characterized by reproductive problems (including stillbirth and fetus mummification), lethargy, cutaneous hyperemia, and cyanosis of the ear in young pigs. Pathologic examination showed typical classical swine fever lesions (Figure). Clinical samples from 2 nonvaccinated herds in 2016 were submitted for laboratory analysis.

PCR showed that these samples were positive for CSFV. Other viral pathogens involved in abortions (e.g., porcine reproductive and respiratory syndrome virus, Aujeszky disease virus, porcine parvovirus, Japanese encephalitis virus, and encephalomyocarditis virus) were not detected in any samples; however, lymph node, tonsil, lung, and brain fetal specimens and placenta specimens from farm $\mathrm{A}$ and lung specimens from farm B were weakly positive for porcine circovirus type 2, which is ubiquitous in South Korea (5). At farm B, serum samples from $20 \%$ of suckling piglets and $30 \%$ of weaned pigs were positive for CSFV. Although blood samples from growing and finishing pigs were not positive for CSFV, fecal samples were positive, indicating possible horizontal transmission in the field.

LOM isolates JJ-1601 (identified in a placenta sample from farm A) and JJ-1602 (in a spleen sample from farm B) shared $99.0 \%$ nucleotide identity with each other; and JJ1601 shared $99.1 \%$ and JJ-1602 shared $99.5 \%$ nucleotide identity with the LOM strain. However, they shared low nucleotide identity (84\%) with $\mathrm{PC} 11 \mathrm{WB}$, a virus isolated from a wild boar in South Korea (6). Phylogenetic analysis indicated that both viruses were classified within subgroup 1.1 (online Technical Appendix Figure 2). Compared with LOM, JJ-1601 contained 5 aa and JJ-1602 10 aa substitutions in the $\mathrm{N}^{\text {pro-E}}$-E region; these substituions are not critical for acquisition of pathogenicity (online Technical Appendix Table 2) (7).

In this study, we observed residual virulence of the LOM strain in naive herds. Since CSFV vaccine was accidentally introduced onto Jeju Island in 2014, continuous LOM outbreaks have occurred (online Technical Appendix Table 3), resulting in tremendous damage to pig farms on the island. In addition, the virus has persistently circulated and caused repeated problems within the infected herds. Given that accidental vaccination was limited in 2014, the

${ }^{1}$ These authors contributed equally to this article. 